\title{
Cannabis use and co-use in tobacco smokers and nonsmokers: prevalence and associations with mental health in a cross-sectional, nationally representative sample ofadults in Great Britain, 2020
}

\author{
Chandni Hindocha ${ }^{1}$, Leonie Brose ${ }^{2}$, Hannah Walsh, Hazel Cheeseman ${ }^{3}$ \\ 1 University College London, University of London \\ 2 King's College London \\ 3 Action on Smoking and Health, ASH
}

Funding: The author(s) received no specific funding for this work.

Potential competing interests: The author(s) declared that no potential competing interests exist.

\section{Abstract}

This study has published https://onlinelibrary.wiley.com/doi/abs/10.1111/add.15381

Background and aims: In Great Britain, cannabis and tobacco are commonly used substances, both independently and together. Use of either substance is associated with mental health problems, but prevalence of co-use within these populations is unknown. We aimed to 1) estimate prevalence of cannabis use, frequency of use and routes of administration (ROA) among tobacco smokers and non-smokers and 2) investigate mental health problems amongst non-users, tobacco-only, cannabis-only and co-users of both substances.

Design: Cross-sectional national online survey (Action on Smoking and Health) fielded in February-March 2020.

Setting: Great Britain

Participants: Adults in Great Britain aged $\geq 18$ year $(n=12,809)$

Measurements: Tobacco use status (smoker [daily or non-daily] or non-smoker [never or ex-smoker]), cannabis use frequency (never to daily), detailed ROAs of cannabis, self-reported treatment for mental health disorders (depression, anxiety, and any). Statistically weighted prevalence estimates were computed to ensure representativeness. Correlates were assessed using chi-squared tests and logistic regression.

Findings: In Great Britain in 2020, 7.1\% of the sample had used cannabis in the past year. Tobacco smokers had greater odds of using cannabis in the past year (21.9\%) and using cannabis daily (8.7\%) than non-smokers (past-year: 4.7\%; aOR=10.07, [95\% Cl: 8.4 -12.0]; daily: 0.7\%; aOR=24.6, [95\% Cl: 17.96-35.55]). Co-administration with tobacco was common (46.2\% of non-smokers, $80.8 \%$ of tobacco smokers). Co-users reported the highest prevalence of any treatment for mental health problems (54.2\%) in comparison to cannabis-only (45.8\%), tobacco-only (33.2\%) and nonusers $(22.7 \%$; all $\mathrm{p} \leq 0.05)$. 


\section{Conclusion:}

Approximately one in 13 adults in Great Britain reports having used cannabis in the past year, approximately four times as many among cigarette smokers as non-smokers. Co-administration of cannabis and tobacco, via smoking, appears to be common, including among self-identified non-smokers. Mental health problems appear to be particularly common among dual users.

\section{Introduction}

Globally, approximately 219 million people use cannabis and 1.1 billion use tobacco'. Two and a half million individuals in England and Wales used cannabis in the last year according to the Independent Review of Drugs 2,3 . Cannabis used increased by $16 \%$ between $2016 / 17$ and $2018 / 19^{2}$ and is now one of the most cited problematic substances amongst those entering substance misuse treatment in the $\mathrm{UK}^{2}$. In Europe, cannabis is the primary drug cited by first-time clients entering substance misuse services, increasing by $76 \%$ in the last decade 4 . In contrast, an estimated 7.7 million UK adults smoke tobacco; prevalence in 2018 was $14.7 \%$. Tobacco smoking remains the leading cause of preventable ill healtth . Both cannabis and tobacco smoking are associated with mental health disorders ${ }^{6-9}$. Estimates suggest $34 \%$ of people with depression and $29 \%$ of people with anxiety in the UK smoke tobacco ${ }^{5}$, both significantly higher than the population prevalence.

Cannabis and tobacco are commonly used together worldwide, by the same individuals, and in the same period ${ }^{0-16}$. In the UK and Europe, it is also common to co-administer cannabis with tobacco in the same product (a joint/spliff). Data from a sample of illicit drug users collected in 2014 suggests $77.2 \%$ of past-year cannabis users in the UK mix cannabis with tobacco ${ }^{11}$. The harms of cannabis are exacerbated by its relationship with tobacco ${ }^{7}$, and co-users are at greater risk of poorer health-related, psychiatric, psychosocial and cessation outcomes for both substances ${ }^{18-20}$.

Whist nationally representative data exist on cannabis and tobacco use individually, data regarding the vast overlap of these substances, and its relationship to mental health, is rare in Great Britain. Our aim was to generate estimates of cannabis prevalence in the population, as well as within tobacco users and non-tobacco users by providing a detailed assessment of frequency of use, routes of administration $(\mathrm{ROA})$ and the prevalence of mental health disorders amongst co-users, exclusive cannabis and tobacco-only groups and non-users.

\section{Methods}

\section{Design and Procedure}

We conducted analyses of data from a cross-sectional, national, online survey carried out in Great Britain between 17th February - 11th March 2020. The survey is commissioned annually by the charity Action on Smoking and Health (ASH) 
and included questions relevant to cannabis and tobacco co-use for the first time in 2019. Selected findings have been published ${ }^{21-23}$. The 2020 survey used a panel of over 1,630,000 UK adults maintained by the market research company YouGov Plc which abides by British Polling Council and ESOMAR (World Association of Opinion and Marketing Research Professionals) guidelines. To represent the national profile of adults over 18 years old (including people without internet access), YouGov statistically weight data by respondents' age, sex, social class, region, level of education, and ethnicity to ensure representativeness in relation to the population. Weights are validated by 3 key sources: 2011 Census; large scale probability surveys; and population estimates from the Office for National Statistics (https://yougov.co.uk/about/panel-methodology/) such that the data is representative of these. The YouGov Panel members were emailed an invitation to participate without information on survey content. YouGov employ an automated sampling system, which allocates respondents to surveys based on quota, and other, requirements. When respondents receive the link, it is not in relation to a specific survey - only when respondents click the link that they are allocated to a specific survey by the system. Of those who started this survey, $97 \%$ completed it. Panel members consent to completing surveys in return for a modest financial incentive. Additional ethical approval was not sought due to this pre-existing consent. Recodes and analyses for the present manuscript were run by the authors using data collected by YouGov.

\section{Sample}

A sample of 12,809 people completed the survey and responses were weighted to be representative of the adult population.

\section{Measures}

Socio-demographics: Age (18-24; 25-34; 35-44; 45-54; 55+), sex (men, women), and socioeconomic status (AB: higher or intermediate managerial, professional and administrative, C1: supervisory, clerical, junior managerial, administration or professional, C2: Skilled manual workers, DE: semi and unskilled manual workers, state pensioners, trainees, never workers and long-term unemployed). Ethnicity (white vs. black and ethnic minority (BAME)), location (England, Scotland or Wales (based on home postcode)), education (Low: no certifications/ GCSEs \& equivalents, Medium: A-levels \& equivalents/ technical qualifications below degree, or High: university degree or above).

Tobacco smoking status: 'Smoking in this survey refers to all burnt tobacco products. It does NOT include e-cigarettes. Which of the following statements BEST applies to you?" Responses were: I have never smoked; I used to smoke but I have given up now; I smoke but I don't smoke every day; I smoke every day. This was treated as "Non-smokers" (never and ex-smokers) and "Smokers" (non-daily and daily smokers) for analysis as the groups did not vary significantly on relevant variables.

Frequency of cannabis use: "In the last 12 months, how often have you used marijuana/ cannabis in ANY WAY?" Responses were: I have never used cannabis; I have used cannabis, but not in the past year; less than once a month; about once a month; about once a week; daily; prefer not to say; don't know. Past-year cannabis users defined as those 
using "less than monthly" or greater for analysis.

Route of administration: "Which option best describes your use of cannabis over the past year?" Responses were: smoked it without tobacco; smoked it with tobacco e.g. (joints/spliffs) or in a blunt (cigar); vaped it in liquid form in an ecigarette or other vaping device; used a vapouriser to heat the leaves or dried plant material; used a vapouriser to heat hash oil; dabbed concentrates such as shatter, budder, or wax; consumed it in food or drinks; some other way; prefer not to say; don't know. Participants ticked the options that applied to them.

Mental health: "The following questions are about your health. We understand that this is a highly sensitive topic and would therefore like to remind you that any information you give is strictly confidential and will be used for research purposes only. Some questions asked may not necessarily apply to you. In the last 12 months, which of the following conditions, if any, have you had any treatment or taken any prescribed medication for? Please select all that apply." Responses were: depression; anxiety; obsessive compulsive disorder; panic disorder or phobia; post-traumatic stress disorder; psychosis; personality disorder; attention deficit hyperactivity disorder; an eating disorder; alcohol misuse or dependence; drug misuse or dependence; problem gambling; none of these; prefer not to say; don't know. Participants ticked all options that applied to them. The last three were exclusive i.e. only available if none of the others were ticked.

\section{Statistical analysis}

In all analyses, data were weighted by age, sex, social class, region and level of education. Prevalence of cannabis use, frequency of use, ROA and mental health outcomes were assessed using valid percentages overall and split by tobacco smoking status. We conducted $2 \times 2$ chi squared $\left(x^{2}\right)$ test of independence to compare the proportion of tobacco smokers and non-smokers across cannabis use frequency categories and to compare prevalence of ROAs of cannabis by tobacco smoking status. Significance was assessed with $p \leq 0.05$. Cramer's $V$ is reported as a measure of the strength of association using traditional thresholds for effect sizes (small: $\mathrm{V}=0.1$, moderate: $\mathrm{V}=0.3$, large: $\mathrm{V}=0.5)^{24}$.

We applied adjusted logistic regression models, controlling for age (18-24, 25-39, 40-49, 50-65, 65+), sex, education (low, mid, high), ethnicity (white vs. BAME), GB location (England, Wales, Scotland) and social grade (AB, C1, C2, DE) to assess the association between tobacco smoking status (smoker vs. non-smoker) and cannabis use frequency status versus never used cannabis (e.g. daily vs never, weekly vs. never). Significance was assessed for all models using Wald test statistics with $\mathrm{p} \leq 0.05$ and by assessing non-overlapping confidence intervals $(\mathrm{Cls})$. No model selection processes were utilized.

As prevalence for treatment for many mental health disorders was low, we restricted our results to the most prevalent responses: "depression", "anxiety" and "none of these" which was inverted into "any mental health problem" i.e. those who said yes to any of the preceding conditions. We used $x^{2}$ tests to compare four groups: non-tobacco smokers and non-cannabis users (non-users), tobacco smokers (who did not use cannabis), cannabis users (who did not use tobacco) and a co-using sample (those who reported being a tobacco smoker and had used cannabis in the past year). Column proportions were compared using Bonferroni-corrected pairwise tests. 
"Don't know" and "prefer not to say" responses were excluded throughout thus some totals do not equal 100\%.

Percentages calculated from cells with under 50 individuals are subject to a larger degree of uncertainty. These results should be considered exploratory, as the analysis presented here was not pre-registered. A preprint of this article before peer review can be accessed online (https://www.qeios.com/read/2F4AQ1 $)^{25}$.

\section{Results}

Overall, $13.9 \%$ (95\% Cl: $13.9-14.9 \%)$ of the sample were tobacco smokers and $43.3 \%$ (40.1-46.5\%) of cannabis users were tobacco smokers. Social grade was numerically comparable across the full sample and the subsample of past-year cannabis users. Visual inspection shows age and gender were skewed towards younger males in the cannabis-using subsample compared to the full sample (Table 1).

\section{Cannabis use among tobacco smokers and non- tobacco smokers}

Overall, $28 \%$ (95\%Cl: $27.2-28.8 \%)$ of respondents had ever used cannabis, $7.1 \%(6.7-7.5 \%)$ had used cannabis in the past year, 3.1\% (2.8-3.4\%) used less than monthly, 3.9\% (3.6-4.2\%) were using at least monthly, 1.2\% (1.0-1.4\%) were using weekly and 1.8\% (1.5-2.0\%) were using daily. Of past-year cannabis users [n=904], 44.5\% (41.3-47.7\%) were using less than once per month, $13.4 \%$ (11.2-15.6\%) were using about monthly, 17.1\% (14.6-19.6\%) were using weekly, and $25 \%(22.2-27.8 \%)$ were using daily (Table 1$)$.

Amongst those identifying as non-tobacco smokers [n=11025], 24.5\% (23.7-25.8\%) reported ever using cannabis of which, 19.9\% (19.2-20.6\%) reported using cannabis, but not in past year. 4.7\% (4.3-5.1\%) reported past-year use, $2 \%$ $(1.7-2.3 \%)$ reported at least monthly use, $1.3 \%(1.1-1.5 \%)$ reported at least weekly use, and $0.7 \%(0.5-0.9 \%)$ were daily users (Table 2).

Within the tobacco smokers [n=1781], 49.8\% (47.5-52.1\%) had ever used cannabis, 27.8\% (25.7 - 29.9\%) had used cannabis, but not in the past year. 21.9\% (20.0-23.8\%) were past-year users, 15.5\% (13.8-17.2\%) were at least monthly users, $13.1 \%$ (10.9-15.3\%) at least weekly users and $8.7 \%$ (7.4-10.0\%) were daily cannabis users (Table 2).

Bivariate associations in Table 2 show there were more tobacco smokers in each cannabis use frequency group than nontobacco smokers. Adjusted odds ratios show that the association between tobacco smoking status and cannabis use frequency became stronger the more frequently cannabis was used. Tobacco smokers have 10.07 ([8.4-12.0]; $p \leq 0.001)$ times greater odds of being a past-year cannabis user in comparison to non-smokers and had 24.6 ([18.0-33.6], $p \leq 0.001$ ) times greater odds of being a daily cannabis user in comparison to non-smokers (Figure 1).

\section{Routes of administration and co-administration}

Of past-year cannabis users [ $\mathrm{n}=904], 75.9 \%$ (73.1-78.7\%) of people reportedat least one method of smoking cannabis, 
either with (61.1\%; [57.9-64.3\%]) or without (27.1\%; [24.2-30.0\%]) tobacco. 21.1\% (18.4-23.8\%) reported having used some type of vaporizer (Table 1). Bivariate associations in Table 3 suggest a significantly greater proportion of tobacco smokers (88.3\%; [85.1-91.5\%]) had used a smoked (combustible) method of administering cannabis than non-smokers (66.5\%; [62.4-70.6\%]). There were opposite patterns with non-smokers who mostly used cannabis without tobacco. A greater proportion of tobacco smokers were co-administrators (Table 3). There was no significant difference between the proportion of people who vaped cannabis overall. More non-tobacco smokers than tobacco smokers were using dry-herb vaporisers, consumed cannabis in food and drink and used alternative methods.

\section{Mental health outcomes (Figure 2)}

Rates of treatment for depression were highest in co-users (32\%; [27.4-36.6\%]) of cannabis and tobacco, comparable across exclusive cannabis-only (18.3\%; [15.0-21.6\%]) and tobacco-only (20.3\%; [18.1-22.5\%]) categories, and lowest in non-users of both substances $\left(12.3 \% ;[11.7-12.9] ; X^{2}(3)=178.91, p \leq 0.001, V=0.12\right)$ with a similar pattern reporting treatment for anxiety $\left(X^{2}(3)=144.82, p \leq 0.001, V=0.11\right.$ ) (Figure 2). For those reporting treatment for "any mental health problem", non-users of both substances reported the lowest percentage (22.7\%; [21.9-23.5\%]), followed by tobacco-only smokers (33.2\%;[30.6-35.8\%]) then cannabis-only users (45.8\%; [41.5-50.1\%]). No significant pairwise difference emerged between the cannabis-only group and the co-using group (54.2\%; [49.3-59.1\%]; $\left.\chi^{2}(3)=361.80, p \leq 0.001, V=0.17\right)$ for treatment for any mental health problem.[1] Prevalence details can be found in Table S1.

\section{Discussion}

This study is the first to examine cannabis and tobacco co-use and its association with mental health problems in a nationally representative sample of over 12,000 adults in Great Britain. The findings indicate higher rates of past-year cannabis use amongst tobacco smokers compared to non-tobacco smokers, higher rates of tobacco use amongst cannabis users compared to non-cannabis users and high rates of daily cannabis use across the sample. We found greater self-reported treatment for mental health problems amongst cannabis users, tobacco users and co-users in comparison to non-users and a significant association between cannabis use and depression is highlighted. This study is one of the first of this scale to use detailed exploration of co-use types, revealing tobacco exposure amongst cannabis users which is often missed in population prevalence surveys ${ }^{16}$ and provides new insights into non-combustible cannabis use.

\section{Tobacco smokers at higher risk of cannabis use}

Tobacco smokers reported past year cannabis use at a much higher rate than non-tobacco smokers and had ten times the odds of past-year cannabis use compared to non-smokers. The association between tobacco smoking status and cannabis use frequency increased across frequency of use. In this sample, tobacco smokers had 24.6 times greater odds of being a daily cannabis user, compared to non-tobacco smokers which aligns with previous research ${ }^{16}$. This survey is not able to determine if the association is causal or the result of common risk factors, however better understanding of this 
relationship would aid strategies to both reduce tobacco and cannabis consumption.

Forty-three percent of past-year cannabis users identified as tobacco smokers, whereas the population level of tobacco smoking in 2018 was $14.7 \%^{5}$, and $13.9 \%$ in this sample. This suggests that tobacco smoking in past-year cannabis users is roughly three times that of the population. This prevalence is comparable to the $44.4 \%$ tobacco smoking prevalence in adults presenting to community treatment for alcohol ${ }^{26}$, although respondents in this study were not necessarily treatmentseeking. Of note, one in four past-year cannabis users reported daily cannabis use; this is far higher than previous estimates suggesting 1 in 10 used daily ${ }^{2,3}$. This suggests daily use may be more prevalent than previously thought and requires careful monitoring in the future since it is associated with greater risk of harms ${ }^{27}$.

\section{Use of non-combustible cannabis}

Use of vaporised cannabis of any form in this study constituted $\sim 20 \%$ of all cannabis use, and was slightly more common in non-tobacco smokers than tobacco smokers, consistent with avoidance of combustible products. Non-tobacco smokers had significantly higher prevalence of using dry-herb vaporisers as well as other alternative routes, whilst tobacco smokers only had a significantly higher prevalence of smoking cannabis with tobacco. Past-year dry-herb vaporisation was the most common type of vaporising (11.6\%). Roughly $9 \%$ had used cannabis in an e-cigarette (liquid form). It is important to monitor the use of cannabis e-liquids given the recent outbreak of 'e-cigarette or vaping associated lung injury' (EVALI) in the US. The UK Medicines and Health Regulatory Agency have only reported two fatal EVALI cases, but 68 deaths occurred in the US ${ }^{28,29}$. Eighty-six percent of these reports included THC and vitamin E acetate has also been identified as a cause ${ }^{28}$. Though it should be noted that Vitamin E acetate is not permitted in e-cigarettes in the UK or the EU, it is important to continue to monitor the chemical composition and prevalence of use of illicit THC e-liquids in the UK. Although it is expected that consumption of cannabis through vaporisers is less harmful than combustible tobacco, understanding this trend in the future is important. Population level statistics on edible cannabis preparations are not available in the UK. However, the rates found in this survey are similar to those seen in the 2017 International Tobacco Control Policy Evaluation Youth Tobacco and Vaping Survey completed in England ${ }^{30}$.

\section{Hidden Tobacco Exposure}

Amongst past-year cannabis users who reported being tobacco smokers, $81 \%$ reported co-administration of both substances, and $90 \%$ reported a combustible form of cannabis use. Of note, nearly half of past-year cannabis users who self-identified as non-tobacco smokers reported co-administering cannabis with tobacco, and $67 \%$ reported a combustible form of cannabis use. Not only are these cannabis users exposed to the harms of combustible consumption, but they are also exposed to the harms of tobacco use, both for the duration of cannabis consumption but also to the risk of developing or maintaining tobacco dependence.

The practise of co-administering cannabis with tobacco, but not identifying as a tobacco user, has been found elsewhere ${ }^{10,30}$. In qualitative studies, young cannabis users ${ }^{31,32}$ describe co-administration as facilitating burning, and this is how they learned to smoke cannabis. However, co-administration can increase the amount of THC uptake ${ }^{33}$. Recent research suggests that UK-based, non-dependent, recreational users of co-administered cannabis and tobacco 
(but who do not necessarily smoke tobacco separately) include about $0.35 \mathrm{~g}$ tobacco per joint, equivalent to roughly one third of a cigarette ${ }^{34}$. This exposes participants to cotinine levels suggestive of moderate tobacco exposure, equivalent to that found in light/moderate cigarette smokers ${ }^{35}$. This is an important observation because development of nicotine dependence symptoms has been observed in occasional (light and intermittent) smokers who do not smoke tobacco daily ${ }^{16,36}$. Co-administration of cannabis and tobacco is not necessarily more acutely rewarding ${ }^{37,38}$, but it does produce more negative acute cardiovascular effects ${ }^{38}$ and is associated with chronic bronchitis even at low exposure ${ }^{39}$. Coadministration is also associated with higher risk of dependence and poorer psychosocial outcomes 40,41 .

Overall, this research suggests there is an additional but hidden level of tobacco exposure in tobacco smokers and nonsmokers who use cannabis, with approximately $81 \%$ and $46 \%$ respectively reporting co-administration. This has implications for population-level estimates of tobacco prevalence. As tobacco use declines in the UK, this raises the possibility of an increasing group in the population exposed to combustible tobacco through cannabis consumption but poorly identified through mainstream surveys. In addition, smoking rates remain higher amongst those in high-risk populations who often experience psychiatric co-morbidities ${ }^{42}$. Since reducing tobacco smoking prevalence remains an important public health goal, cannabis users should be considered a vulnerable population where rates of tobacco smoking are both much higher than in the general population and potentially under-estimated. Use of cannabis and tobacco should be considered concurrently in the development of policy, public health and treatment approaches ${ }^{20}$.

\section{Mental health}

This study found that co-use of tobacco and cannabis was associated with higher rates of both depression and anxiety when compared to single-substance or non-use. When examining treatment for any mental health problem, in addition to a higher prevalence amongst co-users compared to non-users, cannabis use was associated with a significantly higher prevalence compared to tobacco use or no use.

Independently, both cannabis and tobacco use are known to be more common in those with mental health disorders ${ }^{-9}$. When adjusting for pre-birth confounders, the association between cannabis and depression is stronger than that of tobacco and depression ${ }^{43}$. Large scale, longitudinal, epidemiological research from the US suggests that past-month cannabis use is twice as common amongst people with depression than without ${ }^{44}$. Our findings support a significant association between cannabis use and depression, and demonstrate a new finding of a significant relationship between co-use and depression.

As these data are cross-sectional, causality and directionality between tobacco and cannabis use and experience of a mental health problem cannot be inferred, but is it evident there is a close relationship between use, and co-use, of both substances and experience of such a problem. Our findings indicating a high prevalence of co-use suggest that attempts to address either cannabis or tobacco use amongst those with a mental health problem must consider use of the other substance. Evidence on optimising cannabis and/or tobacco dependence treatment in the context of co-use is currently lacking ${ }^{16}$, but it is clear this more vulnerable population requires specific clinical and research attention in tobacco, cannabis and co-use interventions. In addition, impact of use and co-use on mental health treatment outcomes also 
warrants attention.

\section{Limitations}

Limitations of this study include that some estimates are based on relatively small numbers of respondents, any percentage reported based on less than 50 individuals are subject to a larger degree of fluctuation. However, we have included these estimates because these are the first nationally representative data focussed upon Great Britain. We have highlighted where this is the case, and have not made conclusions about these particular statistics. Therefore, they should be considered preliminary. Another potential limitation is the impact of the start of the COVID-19 outbreak, which coincided with data collection (Feb-March 2020), which may have influenced use of tobacco and cannabis in as yet unknown directions.

We acknowledge there is great variation in types of co-use including products which we not able to capture in this analysis, including sequential co-use and amount of use of each substance. Respondents may have misunderstood questions regarding edible cannabis products, as many CBD products utilise cannabis symbology in marketing strategies. However, our estimates seem similar to previous findings ${ }^{30}$. In regard to treatment for mental health problems, the response "none of these", was subsequently inverted into "any mental health problem". Those who responded as "none of these" may have experienced another mental health problem not listed, though the frequency is likely to be low since we covered the most common mental health problems.

\section{Conclusions}

A representative survey of the adult population in Great Britain in 2020 found cannabis and tobacco co-use to be common. Tobacco smokers had ten times the odds of being a past-year cannabis user than non-smokers and 24 times the odds of being a daily cannabis user. Co-users of cannabis and tobacco had the highest prevalence of poor mental health and daily cannabis use compared with those using one or none of these substances.

Acknowledgments: The authors thank Brian Eastwood of Public Health England and Jane Rowe from YouGov for their helpful comments on an earlier version of this manuscript.

\section{$\underline{\text { References }}$}

1. UNODC. World Drug Report 2019. United Nations publication, Sales No. E.19.XI.8, 2019.

2. Black C. Review of Drugs - evidence relating to drug use, supply and effects, including current trends and future 
risks. In: Office H, editor.

https://assets.publishing.service.gov.uk/government/uploads/system/uploads/attachment data/file/868404/Review of Dru gs_Evidence_Pack__FINAL_PUBLICATION_VERSION_1.pdf: Home Office; 2020.

3. ONS OfNS. Drugs Misuse: Findings from the 2018/19 Crime Survey for England and Wales. In: Unit CaPA, editor. London: Home Office; 2019.

4. EMCDDA. European Drug Report 2018: Trends and Developments. Publications Office of the European Union, Luxembourg, 2018.

5. Office for National Statistics. Adult smoking habits in the UK: 2018.

https://www.ons.gov.uk/peoplepopulationandcommunity/healthandsocialcare/healthandlifeexpectancies/bulletins/adultsmo kinghabitsingreatbritain/2018: Office for National Statistics,, 2019.

6. Taylor GMJ, Itani T, Thomas KH, et al. Prescribing Prevalence, Effectiveness, and Mental Health Safety of Smoking Cessation Medicines in Patients With Mental Disorders. Nicotine Tob Res 2020; 22(1): 48-57.

7. Lowe DJE, Sasiadek JD, Coles AS, George TP. Cannabis and mental illness: a review. European archives of psychiatry and clinical neuroscience 2019; 269(1): 107-20.

8. Royal College of Physicians. Smoking and mental health. London: Royal College of Psychiatrists 2013.

9. Lev-Ran S, Feingold D. Chapter 31 - Cannabis Use and Its Association to Mental Illness: A Focus on Mood and Anxiety Disorders. In: Preedy VR, ed. Handbook of Cannabis and Related Pathologies. San Diego: Academic Press; 2017: 298-307.

10. Fix BV, Smith D, O'Connor R, et al. Cannabis use among a nationally representative cross-sectional sample of smokers and non-smokers in the Netherlands: results from the 2015 ITC Netherlands Gold Magic Survey. BMJ Open 2019; 9(3): E024497.

11. Hindocha C, Freeman TP, Ferris JA, Lynskey MT, Winstock AR. No Smoke without Tobacco: A Global Overview of Cannabis and Tobacco Routes of Administration and Their Association with Intention to Quit. Frontiers in Psychiatry 2016; 7(104): 1-9.

12. Hindocha C, Shaban ND, Freeman TP, et al. Associations between cigarette smoking and cannabis dependence: a longitudinal study of young cannabis users in the United Kingdom. Drug Alcohol Depend 2015; 148: 165-71.

13. Schauer GL, Peters EN. Correlates and trends in youth co-use of marijuana and tobacco in the United States, 2005-2014. Drug and Alcohol Dependence 2018; 185: 238-44.

14. Weinberger AH, Pacek LR, Wall MM, et al. Trends in cannabis use disorder by cigarette smoking status in the United States, 2002-2016. Drug and Alcohol Dependence 2018; 191: 45-51. 
15. Goodwin RD, Pacek LR, Copeland J, et al. Trends in Daily Cannabis Use Among Cigarette Smokers: United States, 2002-2014. American Journal of Public Health2017; (0): e1-e6.

16. Hindocha C, McClure EA. Unknown population-level harms of cannabis and tobacco co-use: If you don't measure it, you can't manage it. Addiction In Press.

17. Agrawal A, Budney AJ, Lynskey MT. The co-occurring use and misuse of cannabis and tobacco: a review. Addiction 2012; 107(7): 1221-33.

18. Peters EN, Schwartz RP, Wang S, O'Grady KE, Blanco C. Psychiatric, psychosocial, and physical health correlates of co-occurring cannabis use disorders and nicotine dependence. Drug Alcohol Depend 2014; 134: 228-34.

19. Meier E, Hatsukami DK. A review of the additive health risk of cannabis and tobacco co-use.Drug Alcohol Depend 2016; 166: 6-12.

20. McClure EA, Rabin RA, Lee DC, Hindocha C. Treatment Implications Associated with Cannabis and Tobacco Co-Use. Current Addiction Reports In press.

21. Brose LS, Simonavicius E, Cheeseman H. Awareness and use of'heat-not-burn'tobacco products in Great Britain. Tobacco Regulatory Science 2018; 4(2): 44-50.

22. Simonavicius E, McNeill A, Arnott D, Brose LS. What factors are associated with current smokers using or stopping e-cigarette use? Drug and Alcohol Dependence 2017; 173: 139-43.

23. Eastwood B, East K, Brose LS, et al. Electronic cigarette use in young people in Great Britain 2015-2016Public health 2017; 149: 45-8.

24. Cohen J. Statistical power analysis for the social sciences (2nd Ed.). Hillsdale NJ: Lawrence Erlbaum Associates, Publishers.; 1988.

25. Hindocha C, Brose LS, Walsh H, Cheeseman H. Cannabis use and co-use in tobacco smokers and non-smokers: prevalence and associations with mental health in a nationally representative sample of adults in Great Britain, 2020. Qeios preprint.

26. National Drug Treatment Monitoring System. Substance misuse treatment statistics - National Drug Treatment Monitoring System. ndtms.net: Public Health England, 2018/2019.

27. Englund A, Freeman TP, Murray RM, McGuire P. Can we make cannabis safer?The Lancet Psychiatry 2017; 4(8): 643-8.

28. Medicines and Healthcare product Regulatory Agency. E-cigarette use or vaping: reporting suspected adverse reactions, including lung injury. In: Drug Safety Update, editor. Jan 2020 ed. https://www.gov.uk/drug-safety-update/ecigarette-use-or-vaping-reporting-suspected-adverse-reactions-including-lung-injury; 2020. 
29. CDC. Outbreak of Lung Injury Associated with the Use of E-Cigarette, or Vaping, Products. 2020 (accessed 29th May 2020.

30. Smith DM, Miller C, O'Connor RJ, et al. Modes of delivery in concurrent nicotine and cannabis use ("co-use") among youth: Findings from the International Tobacco Control (ITC) Survey. Substance Abuse 2020: 1-9.

31. Akre C, Bélanger RE, Suris J-C. Cannabis Users Identifying Themselves as Non-Cigarette Smokers: Who Are They? Journal of Child \& Adolescent Substance Abuse2015; 24(6): 331-6.

32. Akre C, Michaud P-A, Berchtold A, Suris J-C. Cannabis and tobacco use: where are the boundaries? A qualitative study on cannabis consumption modes among adolescents. Health Education Research2010; 25(1): 74-82.

33. Van der Kooy F, Pomahacova B, Verpoorte R. Cannabis smoke condensate II: influence of tobacco on tetrahydrocannabinol levels. Inhalation toxicology 2009; 21(2): 87-90.

34. Hindocha C, Freeman, T.P., Curran, H.V. Anatomy of a joint: comparing self-reported and actual dose of cannabis and tobacco in a joint, and how these are influenced by controlled acute administration. Cannabis and Cannabinoid Research 2017; 2(1): 217-23.

35. Bélanger RE, Akre C, Kuntsche E, Gmel G, Suris J-C. Adding tobacco to cannabis-its frequency and likely implications. Nicotine \& Tobacco Research2011; 13(8): 746-50.

36. Shiffman S. Tobacco "chippers"—individual differences in tobacco dependence.Psychopharmacology 1989; 97(4): 539-47.

37. Hindocha C, Lawn W, Freeman TP, Curran HV. Individual and combined effects of cannabis and tobacco on drug reward processing in non-dependent users. Psychopharmacology (Berl) 2017; 234(21): 3153-63.

38. Hindocha C, Freeman TP, Xia JX, Shaban NDC, Curran HV. Acute memory and psychotomimetic effects of cannabis and tobacco both 'joint' and individually: a placebo-controlled trial. Psychological Medicine 2017; 47(15): 270819.

39. Gracie K, Hancox RJ. Cannabis use disorder and the lungs. Addiction 2020; n/a(n/a).

40. Schauer GL, Rosenberry ZR, Peters EN. Marijuana and tobacco co-administration in blunts, spliffs, and mulled cigarettes: A systematic literature review. Addictive Behaviors 2017; 64: 200-11.

41. Agrawal A, Lynskey MT, Madden PA, Pergadia ML, Bucholz KK, Heath AC. Simultaneous cannabis and tobacco use and cannabis-related outcomes in young women. Drug and alcohol dependence 2009; 101(1): 8-12.

42. Taylor G, McNeill A, Girling A, Farley A, Lindson-Hawley N, Aveyard P. Change in mental health after smoking cessation: systematic review and meta-analysis. Bmj 2014; 348: g1151.

43. Gage SH, Hickman M, Heron J, et al. Associations of Cannabis and Cigarette Use with Depression and Anxiety 
at Age 18: Findings from the Avon Longitudinal Study of Parents and Children. PLOS ONE 2015; 10(4): e0122896.

44. Pacek LR, Weinberger AH, Zhu J, Goodwin RD. Rapid increase in the prevalence of cannabis use among people with depression in the United States, 2005-17: the role of differentially changing risk perceptions. Addiction 2020; 115(5): 935-43.

\section{Figures}

Figure 1: Adjusted odds ratio (aOR) of being a tobacco smoker based on cannabis use frequency category vs. never cannabis use. All models adjust for age, gender, education, ethnicity (white vs. BAME), GB location (England, Wales, Scotland) and Social Grade (AB, C1, C2, DE). Error bars represent 95\% Confidence Intervals. All ORs are significant $p \leq 0.05$.

Figure 2: Prevalence of those who reported treatment for mental health disorders (depression, anxiety or 'any mental health problems*) overall and across tobacco and cannabis using groups. Any of these" refers to those selfreporting experiencing: depression, anxiety, obsessive compulsive disorder; panic disorder or phobia; post-traumatic stress disorder; psychosis; personality disorder; attention deficit hyperactivity disorder; an eating disorder; alcohol misuse or dependence; drug misuse or dependence; problem gambling. Prefer not to say and don't know responses not shown. *represents bonferroni-corrected $\mathrm{p}$ value $<0.05$ comparing column proportions after chi-square test.

Table 1: Demographics (gender, age, social grade and tobacco use) of the whole sample and past-year cannabis users.

\begin{tabular}{|c|c|c|c|c|}
\hline $\mathbf{N}$ & $\begin{array}{l}\text { Whole sample } \\
(\mathrm{N}=12809)\end{array}$ & $\%(95 \% \mathrm{Cl})$ & $\begin{array}{l}\text { Past-year cannabis users } \\
(\mathrm{N}=904)\end{array}$ & $\%(95 \% \mathrm{Cl})$ \\
\hline Gender (F) & 6954 & $\begin{array}{l}51.5(50.6- \\
52.4 \%)\end{array}$ & 385 & $\begin{array}{l}42.6(39.4- \\
45.8 \%)\end{array}$ \\
\hline \multicolumn{5}{|l|}{ Age } \\
\hline $18-24$ & 1441 & $\begin{array}{l}11.3(10.8- \\
11.8 \%)\end{array}$ & 242 & $\begin{array}{l}26.8(23.9- \\
29.7 \%)\end{array}$ \\
\hline $25-39$ & 3209 & $\begin{array}{l}25.1(24.3- \\
25.9 \%)\end{array}$ & 386 & $\begin{array}{l}42.7(39.5- \\
45.9 \%)\end{array}$ \\
\hline $40-49$ & 2165 & $\begin{array}{l}16.9(16.3- \\
17.5 \%)\end{array}$ & 140 & $\begin{array}{l}15.5(13.1- \\
17.9 \%)\end{array}$ \\
\hline $50-65$ & 3047 & $\begin{array}{l}23.8(23.1- \\
24.5 \%)\end{array}$ & 90 & $10.0(8.0-12.0 \%)$ \\
\hline $65+$ & 2945 & $\begin{array}{l}23.0(22.3- \\
23.7 \%)\end{array}$ & 46 & $\begin{array}{l}5.10(3.67- \\
6.53 \%)\end{array}$ \\
\hline \multicolumn{5}{|l|}{ Social Grade } \\
\hline$A B$ & 2913 & $\begin{array}{l}22.7(22.0- \\
23.4 \%)\end{array}$ & 216 & $\begin{array}{l}23.9(21.1- \\
26.7 \%)\end{array}$ \\
\hline C1 & 3905 & $\begin{array}{l}30.5(29.7- \\
31.3 \%)\end{array}$ & 305 & $\begin{array}{l}33.7(30.6- \\
36.8 \%)\end{array}$ \\
\hline C2 & 2692 & $\begin{array}{l}21.0(20.3- \\
21.7 \%)\end{array}$ & 148 & $\begin{array}{l}16.3(13.9- \\
18.7 \%)\end{array}$ \\
\hline DE & 3297 & 25.7 (24.9- & 235 & 26.0 \\
\hline
\end{tabular}




\section{Tobacco use}

Never smoked

Ex-smokers

4210

[Non-smokers]

11025

Non-daily smokers

540

Daily smokers

1243

[Smokers]

1783

Cannabis use

Never cannabis use

8805

Ever use but not in the past $12 \mathrm{~m}$

Less than monthly*

Once a month*

121

Once a week*

Daily*

\section{Routes of administration*}

Smoked without tobacco

Smoked it with tobacco e.g. (joints/spliffs) or in a blunt (cigar)

Smoked overall (at least 1 method ${ }^{\circledR}$

2688

402

154

227

245

553

687

Vaped it in liquid form in an e-cigarette or other vaping device

85

Used a vaporiser to heat the leaves or dried plant material 105

Used a vaporiser to heat hash oil

41

Vaped overall (at least 1 method)

191

Dabbed concentrates such as shatter, budder or wax

44

Consumed it in food or drinks

196

Some other way
$53.2(52.3$

$54.1 \%$ )

$32.9(32.1$

$33.7 \%)$

86.1 (85.5-

$86.7 \%)$

$4.20(3.9-4.5 \%) \quad 15$

$9.70(9.2-10.2 \%) \quad 213$

$13.9(13.3$

$14.5 \%)$

$68.7(68.0$

$69.5 \%)$

$21.0(20.3-$

$21.7 \%)$

$3.10(2.80-$

$3.40 \%$ )

$0.90(0.74$

$1.14 \%)$

$1.20(1.01-$

$1.40 \%$ )

$18.0(17.3-$

$18.7 \%$

$1.91(1.67-$

$2.15 \%)$

4.31 (3.96-

$4.67 \%$ )

$5.34(4.95-$

$5.72 \%$ )

$0.66(0.52$

$0.80 \%)$

$0.82(0.66-$

$0.98 \%$ )

$0.32(0.22$

$0.42 \%)$

1.49 (1.28-

$1.70 \%)$

$0.34(0.24-$

$0.44 \%)$

$1.53(1.32$

$1.74 \%)$

$0.51(0.39$

$0.63 \%)$
239

274

513

391

$\%$ *

$44.5(41.3-47.7 \%)$

$13.4(11.2-15.6 \%)$

$17.1(14.6-19.6 \%)$

$25.0(22.1-27.8 \%)$

$27.1(24.2-30.0 \%)$

$61.1(58.0-64.3 \%)$

75.9 (73.1-78.7\%)

$9.4(7.50-11.3 \%)$

$11.6(9.51-13.7 \%)$

$4.6(3.23-5.97 \%)$

$21.1(18.4-23.8 \%)$

$4.9(3.49-6.31 \%)$

$21.7(19.0-24.4 \%)$

$7.2(5.55-8.89 \%)$
$26.4(23.5$

$29.3 \%)$

30.3 (27.3-

$33.3 \%)$

$56.7(53.5-$

$59.9 \%$ )

$16.8(14.4$

$19.2 \%)$

26.5 (23.6-

$29.4 \%)$

$43.3(40.1$

$46.5 \%)$

Notes: $\mathrm{AB}$ - high/intermediate managerial, administrative, or professional; $\mathrm{C} 1$ - Supervisory, clerical and junior 
managerial, administrative or professional; C2 - Skilled manual workers; DE: Semi or unskilled manual workers; E - State pensioners, causal or lowest grade workers, unemployed with state benefits only. " "Smoked overall" and "vaped overall refers to use of at least 1 method, participants could have reported more than 1 within smoked or vaped. * $\mathrm{N}$ is equivalent for total sample and past 12-month cannabis users so only the $\%$ is presented in the past-year cannabis users

Table 2: Cannabis use status by tobacco use status. Bivariate associations represent each cannabis use category vs. never cannabis users

\begin{tabular}{|c|c|c|c|}
\hline & $\begin{array}{l}\text { Non-Smokers } \\
(\mathrm{N}=11025)\end{array}$ & $\begin{array}{l}\text { Smokers } \\
(\mathrm{N}=1781)\end{array}$ & Bivariate association \\
\hline Never used cannabis & 8059 & 746 & \\
\hline$\%$ & $73.1 \%$ & $41.9 \%$ & $X^{2}(1)=588.65, p \leq 0.001, V=0.22$ \\
\hline $95 \% \mathrm{Cl}$ & $72.3-74.0 \%$ & $39.6-44.2 \%$ & \\
\hline Ever used but not in the past $12 \mathrm{~m}$ & 2191 & 496 & \\
\hline$\%$ & $19.9 \%$ & $27.8 \%$ & $X^{2}(1)=213.01, p \leq 0.001, v=0.14$ \\
\hline $95 \% \mathrm{Cl}$ & $19.1-20.6 \%$ & $25.7-29.9 \%$ & \\
\hline Less than monthly & 288 & 114 & \\
\hline$\%$ & $2.6 \%$ & $6.4 \%$ & $X^{2}(1)=179.53, p \leq 0.001 V=0.14$ \\
\hline $95 \% \mathrm{Cl}$ & $2.3-2.9 \%$ & $5.3-7.5 \%$ & \\
\hline Once a month & 78 & 43 & \\
\hline$\%$ & $0.7 \%$ & $2.4 \%$ & $x^{2}(1)=108.50, p \leq 0.001, V=0.11$ \\
\hline $95 \% \mathrm{Cl}$ & $0.5-0.8 \%$ & $1.7-3.1 \%$ & \\
\hline Once a week & 75 & 79 & \\
\hline$\%$ & $0.7 \%$ & $4.5 \%$ & $X^{2}(1)=332.03, p \leq 0.001, V=0.19$ \\
\hline $95 \% \mathrm{Cl}$ & $0.5-0.8 \%$ & $3.5-5.4 \%$ & \\
\hline Daily & 72 & 154 & \\
\hline$\%$ & $0.7 \%$ & $8.7 \%$ & $X^{2}(1)=874.35 p \leq 0.001, V=0.31$ \\
\hline $95 \% \mathrm{Cl}$ & $0.5-0.8 \%$ & $7.4-10 \%$ & \\
\hline
\end{tabular}

Notes: \% represents the number within the tobacco smoker group. Percentages calculated from cells with under 50 individuals are subject to a larger degree of fluctuation. Prefer not to say and don't know responses not shown. 
Table 3: Routes of administration within past-year cannabis users stratified by smoking status. Bivariate associations represents those responding yes vs. no for each route. Data is also reported as a percentage of the whole cannabis-using sample and as a \% of the whole sample.

\section{Route of administration}

Smoked without tobacco

$\%$

$95 \% \mathrm{Cl}$

Smoked it with tobacco e.g. (joints/spliffs) or in a blunt (cigar)

$$
\%
$$

$95 \% \mathrm{C}$

Smoked overall (at least 1 method) ${ }^{\mathrm{a}}$

$\%$

$95 \% \mathrm{Cl}$

Vaped it in liquid form in an e-cigarette or other vaping device

$95 \% \mathrm{Cl}$

Used a vaporiser to heat the leaves or dried plant material

$95 \% \mathrm{Cl}$

Used a vaporiser to heat hash oil

$\%$

$95 \% \mathrm{Cl}$

Vaped overall (at least 1 method)

$\%$

$95 \% \mathrm{Cl}$

\begin{tabular}{l|l|l|}
$\begin{array}{l}\text { Non- } \\
\text { smokers }\end{array}$ & Smokers & \\
& & Bivariate association \\
$(n=513)$ & $(n=391)$ &
\end{tabular}

$26-34 \%$

$27.5 \%$

$237 \quad 316$

$46.2 \% \quad 80.8 \% \quad X^{2}(1)=111.96, p \leq 0.001$,

$41.9-50.5 \% \quad 76.8-84.7 \%$

$341 \quad 346$

$66.5 \% \quad 88.3 \% \quad X 2(1)=57.72, p \leq 0.001, V=0.25$

$62.4-70.6 \% \quad 85.1-91.5 \%$

$45 \quad 30$

$8.8 \% \quad 10.2 \% \quad X 2(1)=0.544 p>0.05$

$6.3-11.3 \% \quad 7.2-13.2 \%$

$78 \quad 27$

$15.2 \% \quad \quad 6.9 \% \quad X^{2}(1)=14.81, p \leq 0.001, V=0.13$

$12.1-18.3 \% \quad 4.4-9.4 \%$

$26 \quad 16$

$5.1 \% \quad 4.1 \% \quad \chi 2(1)=0.489 p>0.05$

$3.2-7 \% \quad 2.1-6.1 \%$

$120-72$

$23.3 \% \quad 18.4 \% \quad X 2(1)=3.301 p=0.069$

$19.6-26.9 \% \quad 14.6-22.2 \%$ 


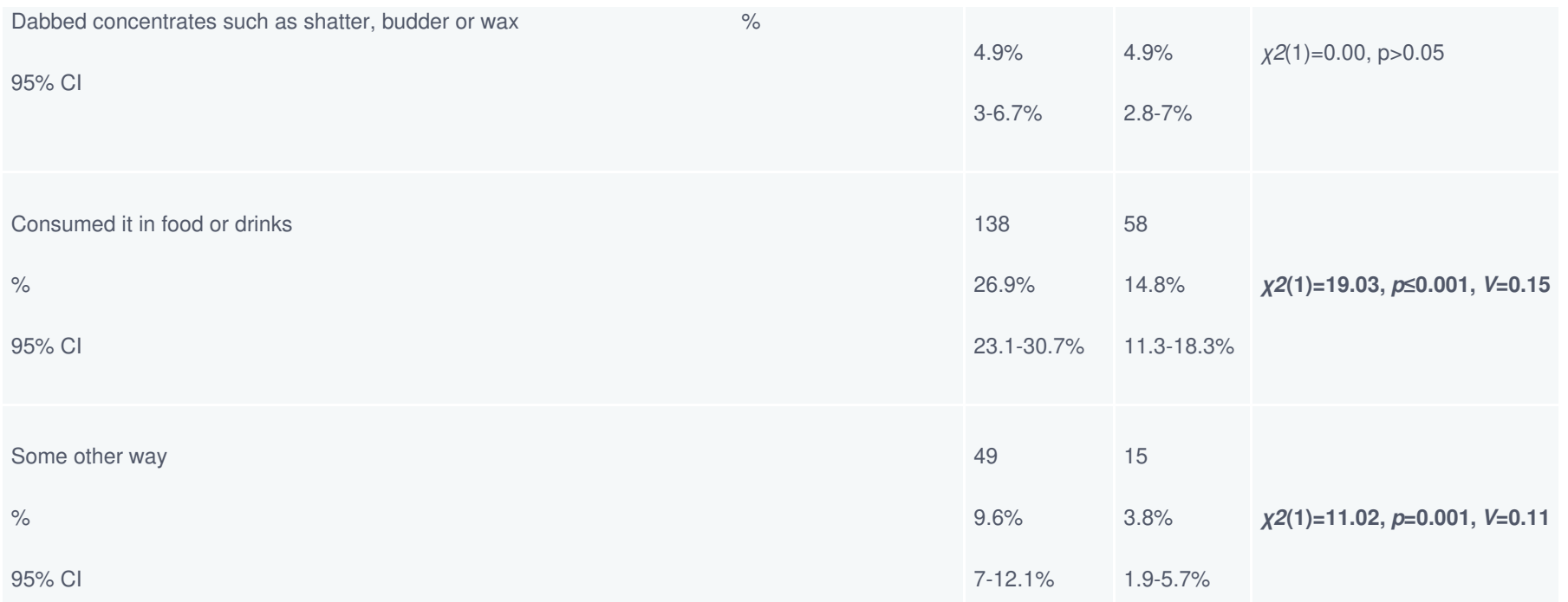

$\%=$ within each smoking status. Percentages calculated from cells with under 50 individuals are subject to a larger degree of fluctuation.Prefer not to say and don't know responses not shown. " "Smoked overall" and "vaped overall refers to use of at least 1 method, participants could have reported more than 1 within smoked or vaped.

[1] Prevalence of treatment for depression, anxiety and "any mental health" problem amongst smokers, regardless of cannabis use status was $22.4 \%, 20.4 \%$ and $40.2 \%$ respectively. Prevalence for depression, anxiety and "any mental health problem amongst past year cannabis use regardless of smoking status was $24.2 \%, 22.5 \%$ and $49.5 \%$, respectively. 